\title{
The unbearable circularity of easy ontology
}

\author{
Jonas $\operatorname{Raab}^{1}$
}

Received: 14 August 2019 / Accepted: 2 November 2020 / Published online: 27 November 2020

(c) The Author(s) 2020

\begin{abstract}
In this paper, I argue that Amie Thomasson's Easy Ontology rests on a vicious circularity that is highly damaging. Easy Ontology invokes the idea of application conditions that give rise to analytic entailments. Such entailments can be used to answer ontological questions easily. I argue that the application conditions for basic terms are only circularly specifiable showing that Thomasson misses her self-set goal of preventing such a circularity. Using this circularity, I go on to show that Easy Ontology as a whole collapses.
\end{abstract}

Keywords Easy ontology · Amie Thomasson - Circularity · Application conditions · Deflationary metaontology

\section{Introduction}

In recent years, Amie Thomasson has been developing a deflationary metaontology called Easy Ontology. The 'Easy' in 'Easy Ontology' is supposed to make obvious that we can answer ontological questions easily - at least if certain conditions are satisfied. Ontological questions are questions asking whether certain entities exist. According to Easy Ontology, those questions are answered by considering whether the corresponding terms refer. The strategy to answer specific ontological questions is to check whether certain terms apply given some uncontroversial truths. This is accomplished by associating terms with what Thomasson calls application conditions. As relations between application conditions give rise to analytic entailments, we only need to check whether some of the uncontroversial truths analytically entail the fulfilment of the application conditions of the terms in question. This is why Easy Ontology is deflationary.

This paper raises doubt whether Thomasson's Easy Ontology is tenable. I argue that it is not-as it is viciously circular. To arrive at this conclusion, I only use what

\footnotetext{
$凶$ Jonas Raab

jonas_raab@web.de

1 University of Manchester, Manchester M13 9PL, UK
} 
Thomasson explicitly endorses as part of Easy Ontology. This is important insofar as there are many charges of begging the question in the debate. For example, Contessa (2016: §2) discusses a charge of begging the question that Thomasson directs towards the fictionalist; he claims that it is rather Thomasson who begs the question. In her reply, Thomasson (2017) claims again that it is the fictionalist who begs the question. ${ }^{1}$ Thus, to prevent the charge of question begging, it is important to use only what she gives us; this shows, then, that Thomasson's Easy Ontology has an internal problemregardless of whether or not it (or an internally consistent version of it, cf. footnote 3 ) is plausible for independent reasons.

The structure of this paper is as follows. In Sect. 2, I present the relevant parts of Easy Ontology as developed by Thomasson. Section 2.1 is a brief account of how Easy Ontology works. Section 2.2 motivates Thomasson's reasons to introduce application conditions and how they are characterized.

Section 3 argues that the account of the application conditions is flawed. Thomasson explicitly imposes a non-circularity condition on application conditions (see (AC2) in Sect. 2.2). I argue that she fails to meet her own requirement. Section 3.1 lays down some ground work and specifies which parts are circular. Section 3.2, then, argues that there is an unavoidable circularity involved. The circularity uncovered in this section is, in certain respects, analogous to definitional circles (more on that below). ${ }^{2}$ Sect. 4 discusses possible objections to my argument.

If the argument of Sect. 3 is correct, then Easy Ontology is circular. Section 5 evaluates how much damage such a charge does to Easy Ontology. Section 6 concludes the paper. ${ }^{3}$

\footnotetext{
${ }^{1}$ Note also Plebani (2018, p. 305f.) who, like me, wants to give a reply to Thomasson that cannot simply be dismissed because it begs the question.

2 Thanks to an anonymous referee for pushing me on making this connection explicit and suggesting the references to Hempel (1952) and Suppes (1957: ch. 8).

${ }^{3}$ Brenner (2018) brings forward an argument that Easy Ontology "will involve an objectionable sort of regress or circularity" (2018, p. 605). As I am likewise arguing that Easy Ontology is circular, let me make explicit two differences between the papers; I will mention more in the course of this paper.

First, Brenner's argument is less general than the one presented in Sect. 3.2 as his only applies to what he calls "terms with non-trivial application conditions" (2018, p. 607). I don't employ such a restriction.

Second, and based on the first point, I think that Brenner (2018) misses the underlying point why such a circularity is damaging. His paper is flawed insofar as it misses out on the core problem for Thomasson's account uncovered in this paper (see Sect. 5).

As far as his paper is concerned, he mentions that "one or more components of Thomasson's so-called easy approach to ontology will need to be modified or abandoned" (2018, p. 605). Both our arguments rely on the deflationary principle (E) and the non-circularity condition (AC2). However, as a response to Brenner's charge, Thomasson could just bite the bullet and accept that those terms with 'non-trivial application conditions' (and presumably only the basic ones among them—see Sect. 3.2 for what 'basic' means here) are circularly specified, and apply her easy approach to all the other terms. Indeed, this is what Thomasson seems to suggest herself (see her 2015, p. 103f., quoted in Sect. 5-interestingly, a passage which Brenner (2018: n. 2 on p. 605), mentions himself but does not discuss).

On the other hand, as I develop the circularity charge and its impact in Sects. 3 and 5, this is not a tenable move for Thomasson because it conflicts with her own account. For, in the light of, e.g., the qua problem (see Sect. 2.2), it is her move to introduce application conditions. These application conditions do the heavy lifting of Easy Ontology because they give rise to the analytic entailments that render the answers to existence questions easy. My argument shows that the approach as a whole collapses because of the way I set the circularity argument up, i.e., it depends on the generality that is lacking in Brenner's paper. In this sense, Brenner's paper does not get to the root of the problem. Indeed, Brenner's (2018) does not even
} 


\section{Thomasson's easy ontology}

\subsection{Easy ontology in a nutshell}

In recent years, Thomasson (2007, 2008, 2009a, b, 2013, 2014, 2015, 2016, 2017) has been developing a deflationary approach to metaontology (we will mainly focus on her $(2007,2015))$. The central idea of the approach is captured in her principle (E):

(E) " $K$ s exist [if, and only if, (iff)] the application conditions actually associated with ' $K$ ' are fulfilled." (2015, p. 86)

In this context, $K$ is a general noun, but as her (2007) witnesses, she thinks that a similar approach can handle the existence of particular objects (see also (2015), n. 1, p. 84). For our discussion the 'actually associated with' part is not important. ${ }^{4}$ Likewise, I only present parts of Easy Ontology that are relevant for this paper and don't attempt to give a full account. ${ }^{5}$

Before further presenting Easy Ontology, let me be explicit about two conventions. First of all, I will follow Thomasson's terminology and speak of 'terms'. 6

Second, let me also be explicit about my usage of 'applies' in the following. ${ }^{7} \mathrm{We}$ can start from a binary version, viz., a term $T$ applies to an entity $e$. This is the case if $e$ is such that it satisfies the application conditions of $T$. Based on this, we can introduce a unary notion and say that a term $T$ applies iff the application conditions of $T$ are fulfilled, i.e., iff there is an entity $e$ such that $T$ applies to $e$. To distinguish this specific version of the unary 'applies', I will call it (E)-applies in the following.

This brings us back to the main discussion. In order to understand principle (E), we need to know what 'application conditions' are and what role they play. In a nutshell, though see Sect. 2.2, they are "conceptual truths" (2015, p. 257f.) that play the crucial role in the answerability of existence questions, viz., "if a term lacks application conditions, we cannot [...] evaluate claims about whether or not the corresponding entities exist" (2015, p. 219). In case a term does have application conditions, we call the corresponding existence question well-formed.

The strategy to answer any particular (well-formed) existence question is to "start from uncontroversial premises" (2015, p. 129) which are such that (i) they do not contain a term $T$, but (ii) they guarantee the satisfaction of $T$ 's application conditions. Since the application conditions are fulfilled, we can use (E) and infer the existence of Ts (cf. 2015, p. 130). Such arguments are called easy arguments.

\footnotetext{
Footnote 3 continued

mention Thomasson's strategy to distinguish between 'basic' and 'derivative' terms in order to make her non-circularity condition (AC2) work.

So, by and large, the argument developed here is more general and has a different scope insofar as it shows the underlying flaw of Easy Ontology. This is also the reason for this paper's title; the circularity is 'unbearable' as it hinders a fix that doesn't undermine the whole easy approach.

4 See her (2015, p. 85f.) for the motivation.

5 Most notably, this paper will not be concerned with what Thomasson calls 'frame-level coapplication conditions' or simply 'coapplication conditions'.

${ }^{6}$ Her specific reason to speak of 'terms' is the following: "I prefer to avoid controversies about concepts, their existence, and nature, by speaking of terms and their application conditions" (2015, p. 85).

7 Thanks to an anonymous referee to pointing out that my use of 'applies' needs disambiguation.
} 
As an example, consider the following easy argument for the existence of events:

[W]e may start from the undisputed truth that May was born on a Monday, and conclude that a birth (an event) occurred on a Monday, and thus that there are events. (2015, p. 130)

Let me be explicit about the steps taken in the argument (but I'll brush over some details such as different tense). We start with 'May was born on a Monday' which is declared an 'undisputed truth' and can therefore serve as starting-point of an easy argument. In order to conclude 'a birth occurred', we need to consider the application conditions of 'birth'. Anticipating the details from Sect. 2.2, we take the conditional 'If someone was born on a Monday, then a birth occurred on Monday' to provide (sufficient) application conditions for 'birth' (where I take 'a birth occurred' to mean the same as 'there is a birth'). Since the uncontroversial truth guarantees the satisfaction of the antecedent of this conditional, i.e., that the application conditions of 'birth' are fulfilled, we can use (E) and conclude that there are births (on Monday), i.e., there is an entity which is a birth. To conclude from that there are events, we have to consider (sufficient) application conditions of 'event' which we take to be 'If something is a birth, it is an event'. Therefore, since we already established that there are births, we can, once more, use (E) and conclude that events exist.

\subsection{Application conditions and the Qua problem}

Thomasson's main motivation for the association of terms with application conditions is that she thinks this move solves the so-called qua problem. The qua problem is a problem for (purely) causal theories of reference (see Devitt and Sterelny 1999). Such purely causal theories need the grounding of reference to be causal in nature, too, such as in what Kripke (1972, p. 96) calls 'baptism'. Thomasson summarizes the qua problem as follows.

But the qua problem arises once we acknowledge that there are terms of many different sorts that at least purport to refer to many different sorts of things, for example, artifacts, lumps of matter, spatial or temporal parts of objects, events, and so on. For those attempting to ground the reference of a new singular term, it will be radically indeterminate what the term refers to (or even whether or not it refers) unless they have some very basic concept of what sort of thing (broadly speaking) they intend to refer to, if the reference grounding is to succeed. (2007, p. 38)

This means that if we progress purely causally, it is indeterminate to what exactly newly introduced terms refer. The solution to this problem is to disambiguate: "nominative terms must be associated with a sortal or, more generally, categorial concept" (2007, p. 39 , my emphasis). If done correctly, this association is, among other things, such that "it must establish certain very basic conditions under which the attempted grounding would or would not be successful in establishing reference" (2007, p. 39)-and these conditions are what Thomasson calls 'frame-level application conditions'. Thomasson also explains the 'frame-level' in 'frame-level application conditions': "they involve 
conditions that are conceptually relevant to whether or not reference is established, not all the conditions that may be empirically discovered as relevant" (2007, p. 39f., her emphasis).

Thus, application conditions derive their plausibility from solving the qua problem, i.e., from the role they play in disambiguating reference-or, more strongly, in even establishing successful reference. This will be important in the following.

Thomasson characterizes further features of application conditions. For our purposes, only one of the three features is relevant:

$\mathrm{AC} 1$ "They are semantic rules of use which speakers master, but these rules needn't take the form of necessary and sufficient conditions, and needn't be statable." (2015, p. 91)

Crucially, Thomasson also adds the following condition:

AC2 "Application conditions must not take the following form: ' $K$ ' applies iff $K \mathrm{~s}$ exist." (2015, p. 96, my emphasis)

Let us briefly consider these in turn. (AC1) is related to a point Thomasson already emphasized in her (2007, p. 44), viz., the association with application conditions does not mean that every competent speaker is in (explicit) possession of them. Indeed, we are rarely capable of explicitly giving the application conditions of any term we use. To make this claim further plausible, she notes that the "history of philosophical attempts" to provide any such is "a history of failures" (2015, p. 91); even philosophers who have tried and those who are still trying have-according to Thomasson-not much to show for. However, Thomasson insists that just because we cannot state them doesn't mean that our terms don't have them [see (2015, p. 92)].

Let me flag, at this point, that there is a certain ambiguity with respect to 'needn't be statable'. We will come back to this in Sect. 4.1.

Feature (AC1) and its motivation also make it difficult to explicitly provide any application conditions. Indeed, Thomasson says:

One thing I should be upfront about, however, is that it is not always perfectly clear what precisely the constitutive rules are that govern any particular natural language expression. (2015, p. 248)

Put differently, it is not perfectly clear what the application conditions of our terms are. This is why I am, likewise, not in a position to provide concrete examples; in Sect. 3.2 I will use the mathematical terms 'set' and 'function' as proxies for fitting examples of terms for which we can provide application conditions. The closest Thomasson comes to stating application conditions is that it is "in part constitutive of the meaning of 'house' that all houses are buildings" (2007, p. 28). Another example is this: A "term like 'marriage' may be introduced and partially governed by the rule 'If two suitable people A and B visit the justice of the peace and fill in the relevant paperwork and say the relevant vows, then A and B got married"' (2015, p. 101). ${ }^{8}$ What these

\footnotetext{
8 As per (AC3) (p. 7), the term 'marriage' needs to be introduced by a conditional providing sufficient conditions for its use. An anonymous referee pointed out that, in the example, the term does not even occur in the conditional. I leave it open how Thomasson wants to align this with her own account. Also, it is not clear whether you can non-circularly spell out the 'suitable' or 'relevant' in the antecedent.
} 
examples have in common is that the (partial) application conditions involve other terms (but see Sect. 4.2).

Moreover, since any explicit statement of application conditions of a term $T$ must contain other terms, some terms must be "semantically basic terms" (2015, p. 92) since, otherwise, we run into a regress or circle (not unlike a definitional circle; see below). Thus, there must be other means to learn how to properly use at least some terms, for example, "ostensively as we learn that a term is to be applied in situations like this (and not in situations like that), or via judgments of similarity to ostended paradigms" (2015, p. 92, her emphases). For now, let us grant her this point. Note, however, that without disambiguation it is not clear what the ostension ostends to; thus, as it stands, we have no reason to not apply Thomasson's own reasoning with respect to the need to introduce application conditions to this case. We return to this in Sects. 4 and 5.

Condition (AC2) is the crucial non-circularity condition. If the only way to determine whether or not a term (E)-applies is by checking whether or not the corresponding entity exists, we would run in a circle and would not be able to deflate our metaontology. The burden on Thomasson is, thus, to show that it is possible to "make it evident that application conditions for ' $K$ ' need not appeal to the existence of $K$ s" (2015, p. 97). Her strategy is "to show a way in which they may be stated without appealing to the existence of $K \mathrm{~s}$ - or of some thing that meets the application conditions for ' $K$ '” (2015, p. 97, her emphasis). To accomplish this, she "divide[s] nominative terms into two cases: concrete nouns of what Carnap called the 'thing' language [...], and derivative nouns [...] that may be introduced on the basis of these" (2015, p. 99). I argue in Sect. 3 that Thomasson is not successful in preventing the circularity and evaluate which damage this does to her approach in Sect. 5. Section 4 discusses several of Thomasson's strategies to prevent the circle.

Note that Thomasson formulates (AC2) with a biconditional, viz., " $K$ ” applies iff $K$ s exist'. To anticipate my understanding of application conditions provided below, I understand the biconditional as follows: I take a condition $\varphi(x)$ to be (sufficient) application conditions of a term $T$ if the condition's satisfaction guarantees the existence of a $T(\varphi(x) \rightarrow T(x))$, and, if to be a $T$ is exhausted by this condition, then it's also necessary $(T(x) \rightarrow \varphi(x))$. As it is not important for our concerns, I won't spell out a tight connection between such conditions and terms, as we'd might want to distinguish $\varphi$ from logically equivalent conditions which don't add anything (such as $\varphi \wedge(\psi \vee \neg \psi)) .^{9}$

Put together, $(\mathrm{AC} 1)$ and $(\mathrm{AC} 2)$ are not unsimilar to what is usually required of definitions. ${ }^{10}$ Just as (AC2) is a non-circularity condition, definitions, too, are not allowed to be circular. (AC1) is, of course, not meant as enforcing the semantic rules of use to take the form of full-fledged definitions, but we can understand the condition as at least enforcing these rules to "give us (e.g.) an open list of sufficient conditions for

\footnotetext{
9 Thanks to an anonymous referee for pointing out this difficulty.

10 For a standard introduction to the topic of theory of definition, see Suppes (1957: ch. 8). Another criterion of definition is the criterion of non-creativity (Suppes 1957, p. 154f.) which is usually guaranteed by the requirement that definitions need to be conservative. Thomasson (2015, p. 263f.) also invokes a conservativeness requirements for the proper introduction of new terms. In my (2020), I argue that this requirement is problematic for Easy Ontology.
} 
the application of" (Thomasson 2015, p. 101) a term; indeed, Thomasson repeatedly speaks of 'sufficient condition for the application of a term' [see, e.g., (2015, pp. 99, $101,107)]$. Whether or not all sufficient conditions of a term jointly build up to a definition is not important.

Moreover, just as we distinguish between primitive and derivative vocabulary in the case of definitions, Thomasson divides the terms into basic and derivative. ${ }^{11}$ Hempel (1952, p. 15) is also explicit that we have to divide the vocabulary into primitive and derivative; one reason to do so is to avoid circular definitions. This is a well-motivated and plausible move in the case of definitions. However, as I will argue, the same strategy does not work for Easy Ontology. On the contrary, Easy Ontology ends up in a vicious circle not unlike one of circular definitions.

Lastly, Thomasson also gives us some information regarding how to introduce new terms. I restrict the presentation to the condition which is relevant in the following. ${ }^{12}$ In order for terms to be "minimally introduced to an unextended language $L$ " (2015, p. 263), the following condition needs to be satisfied:

AC3 “The term(s) must be introduced via a conditional that gives sufficient conditions for its(/their) application" (2015, p. 263).

This means that we introduce a term $T$ by providing a conditional whose antecedent 'gives sufficient conditions'. I take it that the consequent of such a conditional is just the term that's being introduced. Thus, to introduce a term $T$, we need to provide a conditional of the form ' $\varphi(x) \rightarrow T(x)$ ' where ' $\varphi(x)$ ' provides sufficient conditions formulated in terms of the unextended language (or an extended language in which all the terms introduced have been properly introduced). Since the application conditions are supposed to be conceptual truths (2015, p. 257f.), this must mean that the conditional is what's being referred to as 'application conditions'. Since it is supposed to be a conceptual truth that whenever the sufficient application conditions of a term $T$ are satisfied, then this term (E)-applies, I take the application conditions to be the universal closure of such conditionals, i.e., to be of the form ' $\forall x(\varphi(x) \rightarrow T(x)){ }^{\prime} .{ }^{13}$ Such conditionals together with 'uncontroversial premises' give us enough resources to infer a positive instance of $T$. For example, if houses suffice for the application of the term 'Building', i.e., ' $\forall x$ (House $(x) \rightarrow \operatorname{Building}(x))$ ' is (conceptually) true, then using the uncontroversial premise that I am inside a house provides us with the necessary information to infer that there are buildings.

Before moving on, let me introduce another convention: In the following, I will use 'application conditions' to mean the antecedent of such conditionals. With this convention in place, the application conditions of a term $T$ are circular if they essentially contain $T$, so that the only way for the application conditions to be satisfied is by $T$ (E)-applying. Reformulated in terms of conditionals, we have a circularity if,

\footnotetext{
11 As both Hempel (1952) and Suppes (1957) make obvious, definitions do not have to be of the genusand-differentia form - and neither do application conditions.

12 Thomasson (2015, p. 263f.) provides three conditions to introduce a new term $T$ to a language. The second condition is a conservativeness condition which I argue in my (2020) is problematic for her account. See my (2020: §2.4) for an explication of 'analytic entailment' and a discussion of the term introducing mechanisms; see also Sect. 5 .

13 Cf. what Carnap (1952) calls 'meaning postulates'.
} 
for example, the conditional has the form ' $T(x) \wedge \varphi(x) \rightarrow T(x)$ ' where ' $\varphi(x)$ ' might be "empty", i.e., either vacuously satisfied or, understood as a list of conditions, the empty list.

\section{The circularity of the application conditions}

In the following, I will first delineate the scope of the circularity charge in Sect. 3.1 before giving the argument in Sect. 3.2. I will discuss potential objections to such a circularity charge in Sect. 4. Section 4.1 discusses the alleged unstatability of the application conditions before Sect. 4.2 discusses the proposal to circumvent the circularity by supplying application conditions in a different language. As we have just noted, the circularity charge is not unlike the problem of circular definitions and trying to provide definitions for all relevant parts of one's vocabulary. One common suggestion to get around this problem is to introduce an infinite regress; Sect. 4.3 discusses, among others, such an option.

\subsection{Preliminaries and scope of the circularity charge}

Before coming to the circularity charge, note, first, that it is explicitly Thomasson's strategy to divide the terms into 'derivative' and 'basic' in order to show that the noncircularity condition (AC2) holds. As this is the only strategy she mentions, showing it to fail suffices to show that Easy Ontology_as it stands-is circular.

Moreover, since the answerability of existence questions is interlinked with the availability of application conditions (see, e.g., her 2009b), an Easy Ontologist has to provide a new strategy to specify them non-circularly. As Easy Ontology rests on the idea that the triviality of inferences to answer existence questions is due to analytic entailments-which, in turn, rest on the application conditions-there seems to be no room to answer the circularity charge without giving up substantial parts of Easy Ontology. In particular, giving up the analytic entailments that provide reasons for the 'Easy' in 'Easy Ontology', some existence questions cannot be answered easily; hard ontology is back on the table. I argue in Sect. 5 that this problem is more widespread than Thomasson (2015, p. 104) acknowledges; to arrive at this conclusion, we need the circularity argument to be quite general in order to see that it does not depend on how exactly the line between 'basic' and 'derivative' terms is drawn. ${ }^{14}$

Before giving the argument, let us consider one strategy to accomplish a noncircular specification of application conditions - to dismiss it right away. By showing how this strategy fails, we get a better grip on what it takes for application conditions to be non-circular and to see in what sense Thomasson's account does not satisfy it.

Consider a term $T$. As it is often difficult to come up with application conditions for any such term (cf. the discussion of (AC1) in Sect. 2.2), let's introduce another term $T^{\prime}$ and stipulate that the application conditions of ' $T$ ' are that they are $T^{\prime}$ s

\footnotetext{
14 This, in particular, is where my paper is more substantial than Brenner's (2018). Sections 4 and 5 show that Thomasson cannot just bite the bullet and accept that some terms have circular application conditions or even lack any application conditions.
} 
and the application conditions of ' $T$ ' ' are that they are $T \mathrm{~s}$. In this way, it seems as though we satisfy the non-circularity condition (AC2); for we arrive at " $T$ ' (E)applies iff $T$ 's exist” (or “ ' $T$ ' (E)-applies iff ' $T$ ', (E)-applies”). But as we also arrive at " $T$ ' $T^{\prime}$ (E)-applies iff $T$ s exist", we have made no progress. This is also true if the application conditions of at least one of these terms involve other terms; the circularity is more complex then, but still there. For, the only thing that changes is the addition of conditions. But in this case, too, to see whether ' $T$ ' (E)-applies, means, inter alia, to check whether there are $T^{\prime} \mathrm{s}$; and to check whether there are $T^{\prime} \mathrm{s}$, means to check whether the term ' $T$ ', (E)-applies; this, then, means to check whether there are $T$ s, and we have gone full circle. Thus, we should assume obvious restrictions on the proper satisfaction of (AC2) to dismiss such cases.

We can also note that whatever term $T$ we choose, the equivalence " $T$ ' (E)-applies iff $T$ s exist" must be true. ${ }^{15}$ It is only problematic if this is the only available way to specify the application conditions for the respective term. My argument below amounts to the claim that for (at least some) basic terms, this must be the case; therefore, the account is circular in the sense that condition (AC2) is not satisfied. As this is a condition that Thomasson explicitly endorses, my argument shows that her account is insufficient by her own lights. ${ }^{16}$ However, it is not just that there are circular terms which we could isolate and dismiss; rather, as argued for in Sects. 4 and 5, all other terms rely on those circular ones; and this means that the circularity infects the whole approach. In particular, we can see that the problem repeats itself if we choose to dismiss the circular terms; we can apply the same argument again to show that there must be circularly specified terms among the remaining ones-the problem does not go away.

Let me also point out that this boils essentially down to a problem regarding the grounding of reference. If all derivative terms are only insofar disambiguated as they are provided with application conditions, they are only insofar disambiguated as the terms involved in their application conditions are disambiguated - to be disambiguated, as we have seen in Sect. 2.2, just means to be associated with application conditions [see also Thomasson (2007, p. 41, 2015, p. 94)]. My argument shows that the basic terms either have no application conditions or only have circular ones. Both

\footnotetext{
15 Interestingly, Thomasson, when she formulates condition (AC2), immediately says the following in parentheses: "While this [the equivalence " $T$ ' (E)-applies iff $T$ s exist"] will always be true, it will not count as an application condition, in our terms" (2015, p. 96). In the following, I will be more charitable and consider conditions which violate (AC2) to be application conditions because they play an important role in the grounding of reference as outlined in Sect. 2.2; I don't think that there is any room on giving up on this without doing more harm than good. This will become clear in the following. (This takes effectively also care of the suggestion to take certain terms to be primitive terms; they wouldn't have proper application conditions and couldn't give rise to analytic entailments; see Sect. 5.)

16 Note that Brenner's (2018) does not even mention Thomasson's strategy to distinguish between 'basic' and 'derivative' terms in order to make her non-circularity condition (AC2) work. Nor does Brenner make the point that the analytic entailments are endangered by a general circularity charge. His paper leaves it open whether or not an easy approach is viable for terms that are not among those he characterizes as 'terms with non-trivial application conditions'. I will formulate my argument below in terms of the basic terms - which are among the 'terms with non-trivial application conditions' if understood as Thomasson (2015, p. 104) introduces them-but it is clear that this generalizes to any term; this is also suggested by the examples involving mathematical terms which are presented after the presentation of the abstract argument.
} 
options mean that there cannot be disambiguation after all, i.e., the qua problem is not resolved. This is a problem deep down in the approach.

\subsection{The circularity charge}

This brings us to the argument. Recall that Thomasson has to argue that the application conditions for a term ' $T$ ' do not include-in the relevant sense, see Sect. 3.1- the requirement that $T$ s exist (in the following, I will use ' $A C(T)$ ' as shorthand for 'application conditions of term $T$ ' which captures the antecedent of the corresponding conditional as per (AC3)). To this end, she proceeds in two steps. First, she considers 'derivative nouns' (2015, pp. 99-103) which are introduced by explicitly using other nouns. Second, she considers 'basic nouns' (2015, pp. 103-112) which are not so introduced. As the circularity charge is directed towards the latter, the former are irrelevant for the present purposes.

In the following, I first present the abstract argument before spelling it out in a more concrete way. As we already saw that it is difficult to come up with application conditions for any term (recall the discussion of (AC1) in Sect. 2.2), I will use mathematical terms as illustration in the concrete example even though this does not fit the characterization of 'basic' (see below). However, as, in contrast to many other terms, we can even provide definitions for mathematical terms, this clarifies the case at hand. Besides being clarificatory in this sense, this also illustrates that the argument is invariant with respect to the characterization of 'basic' in 'basic term'.

First, we have to understand in what sense the basic terms are basic (but, note again, that even if we draw the line differently, the following argument is not affected). Thomasson considers the following to be the "relevant" (2015, p. 105) sense of "basic term'. Basic terms are terms

we tend to learn early in our cognitive or linguistic development, and that we make use of in acquiring other concepts and learning to use other terms[.] (2015, p. 104)

Following Carnap, she suggests 'piece of paper' and 'desk' as basic terms of 'the thing language', and claims that terms 'of this category, such as 'dog', 'cup', or 'teddy', tend to figure prominently in early language acquisition" (2015, p. 106). She thinks that this supports the claim that "these sortals are at least relatively fundamental" (2015, p. 106).

Moreover, Thomasson claims that

we can show that one may answer an existence question 'Do $K$ s exist?' easily, by appeal to whether the application conditions for ' $K$ ' are fulfilled, if we can show a way to state and understand the application conditions for ' $K$ ' that does not appeal to $K$ s. (2015, p. 106)

As an example, Thomasson uses 'cup': “if there are particles arranged cupwise, we are entitled to infer 'there is a cupwise arrangement of particles', and so to infer: 'there is a cup"” (2015, p. 106f.). 
This is problematic, though. By assumption, 'cup' is a basic term. We have, in general, two options in supplying application conditions for such a term. One option is to supply application conditions exclusively via basic terms; another is to also mix in derivative terms. Both options run into a circularity problem.

As 'particles arranged cupwise' seems not to be a basic term, let us first assume that it is derivative. Then, if the above quotation does indeed give the application conditions of 'cup', we have to go through derivative terms and the existence of their corresponding entities to infer the existence of an entity falling under a basic term. This might not (yet) be problematic in itself. For we have again two options. First, the application conditions of 'particles arranged cupwise' (and of any term that is part of the application conditions of a basic term) might not include reference to cups (and the like). Second, the application condition of 'particles arranged cupwise' do include reference to cups.

As we can dismiss the latter option right away-it is obviously circular (see Sect. 3.1) - let us consider the former option. Suppose that there is a basic term $T$ such that

$$
A C(\text { particles arranged cupwise })=\varphi(T) .{ }^{17}
$$

This, then, means that the application conditions of 'cup' are specified in terms of a term $T$ which does not refer to cups. The question, now, is what the application conditions of $T$ are. We can apply the same reasoning again to conclude that even if all basic terms $T$ can have application conditions like 'particles arranged $T$-wise' where the latter are derivative, we have made no progress as we need to specify the application conditions of the terms 'particles arranged $T$-wise'. These, in turn, can involve derivative terms. In this case, we need to do further work until we reach a specification fully in terms of basic terms. And, as we want to give application conditions for 'cup', none of the terms involved in the application conditions of 'particles arranged cupwise' can be the term 'cup', as this would obviously be circular.

Let us suppose that we have $n$ distinct basic terms; let them be $T_{1}, \ldots, T_{n-1}$, and cup. Then, the application conditions of 'cup' can be a combination of $T_{1}, \ldots, T_{n-1}$, i.e.,

$$
A C(\operatorname{cup})=\psi\left(T_{1}, \ldots, T_{n-1}\right) .
$$

The question, then, is: What are the application conditions of term $T_{n-1}$ ?

On pain of circularity, 'cup' cannot be among them. For, if 'cup' is among them, we end up specifying the application conditions of 'cup' in terms of $T_{1}, \ldots, T_{n-1}$, and the application conditions of $T_{n-1}$ in terms of 'cup'. So, we arrive at the situation we already dismissed in Sect. 3.1; this is so because, by assumption, the current terms are basic. Assuming the application conditions of these terms (here: $T_{n-1}$ and 'cup') to be specifiable in terms of common basic terms (here: $T_{1}, \ldots, T_{n-2}$ ) means that they

17 In principle, the application conditions of 'particles arranged cupwise' can be a combination of many (basic) terms. As nothing changes in the reasoning, I will only consider the case in which they are exhausted by just one. So, in our context, ' $\varphi(T)$ ' just is ' $T$ s exist' (or something similar). 
are not basic, but derivative. ${ }^{18}$ Nonetheless, the application conditions of term $T_{n-1}$ must be a combination of the terms $T_{1}, \ldots, T_{n-2}$, i.e.,

$$
A C\left(T_{n-1}\right)=\vartheta\left(T_{1}, \ldots, T_{n-2}\right)
$$

Following this line of reasoning, we end up with the question: What are the application conditions of the term $T_{1}$ ? As we have used up all the basic terms, there is no way of specifying them non-circularly.

Of course, we might not need all of the other terms to specify the application conditions of one term. We might, for example, only use the term $T_{n-2}$ in the application conditions of 'cup', and only $T_{n-3}$ in the application conditions of $T_{n-2}$, and so on. However, following this kind of reasoning, we still end up with at least one basic term which is used to specify the application conditions of the other terms but whose application conditions are not satisfying the non-circularity condition (AC2) - and exactly those problematic terms are the ones providing non-circular application conditions for all the other basic terms.

In particular, consider the case of exactly one basic term, i.e., let $n=1$. As there is only one basic term, its application conditions_-assuming them to exist-must be circular. As every other term is specified on the basis of the basic terms - in the case at hand just the one-we cannot just use a derivative term in the application conditions of the basic term; for the derivative terms are introduced "on the basis" (2015, p. 99) of the basic terms. ${ }^{19}$ But, since we need terms to have application conditions to circumvent the qua problem, we cannot just assume some terms without. For, this would also destroy the analytic relationships that do the heavy lifting of Easy Ontology with respect to existence questions (see Sect. 5).

To sum this case up, we either end up in a situation that is exactly what condition (AC2) explicitly dismisses, viz., we have a basic term $T$ such that its application conditions are " $T$ ' applies iff $T$ s exist", or we end up in a situation such that we have terms $T_{1}, \ldots, T_{n}(n \in \mathbb{N})$ such that $A C\left(T_{1}\right)=\varphi_{1}\left(T_{2}\right), A C\left(T_{2}\right)=\varphi_{2}\left(T_{3}\right), \ldots$, $A C\left(T_{n-1}\right)=\varphi_{n-1}\left(T_{n}\right)$, and $A C\left(T_{n}\right)=\varphi_{n}\left(T_{1}\right)$, i.e., another circle.

The case in which 'particles arranged cupwise' is itself a basic term is analogous to the one above. As we run out of options, either this term itself is violating (AC2) or there is another term whose application conditions are circular.

By and large, this consideration shows that Thomasson has merely hidden the circularity that she wanted to prevent. The non-circularity condition (AC2) is not

\footnotetext{
18 Note that there is a potential ambiguity in 'basic' and 'derivative'. In principle, a basic term (in the sense of being acquired early in ones development) might still be derivative (in the sense of being specifiable in other terms). However, the argument brought forward in the main body of the text stays the same. In either case we have to wonder what the application conditions are. And the picture Thomasson draws suggests that we start with terms that are semantically basic and use those to introduce further terms. But the easy approach has to apply to such basic terms as well—and this is the root of the problem.

19 The problem here goes even deeper. Thomasson has to place restrictions on terms being "minimally introduced" (2015, p. 263). One such restriction is that only the "extant terms of" $(2015$, p. 263) a base language are allowed to be used; see Sect. 5 for the full quotation. But given that our base language consists of only $n=1$ term, there simply are no other terms to supply application conditions, i.e., we can't even introduce the derivative terms in order to supply application conditions of the basic one. I discuss this more fully in my (2020).
} 
satisfied across the board. In particular, as the terms for which condition (AC2) is not satisfied supply the application conditions of all other terms, this problem affects all terms; see Sect. 5.

We can put the circularity charge in terms of 'reference grounding'. For, according to Thomasson, to have successful reference grounding (which we have to have to circumvent the qua problem), we need what she calls categorial terms. These, in turn, must refer. If they did not refer, there would be no way to use them to successfully ground reference. The reason is simply that we apply further conditions on the categorial term to successfully ground reference to something more specific. For example, assuming 'animal' to be a categorial term, we can use it to introduce the term 'human being' via specifying what kind of animal a human being is supposed to be; traditionally, this would either be 'rational animal' or 'two-footed animal' or even 'social animal'. But, if 'animal' does not (E)-apply at all, it cannot be used to successfully establish reference to anything (any subset of an empty set is itself empty).

Thus, before the reference grounding can even start, we must assume there to be successfully referring categorial terms. These, then, play the role of the basic terms whose application conditions cannot be non-circular. For, there are just no other terms in which they could be specified. But if these terms do not have application conditions, they cannot be used to provide analytic entailments between the terms which are based on them. For, these terms inherit their analytic interrelations from the respective application conditions. But if we start out with terms which do not provide analytic entailments between the terms, the easy approach as a whole collapses (see Sect. 5).

This also shows a mismatch in the approach. To be in a position to introduce all other terms on the basis of basic terms, we need an appropriate set of basic terms. Depending on the choice of such a set, we end up in the following dilemma: Either (i) we assume abstracta (i.e., instances of what is called 'highly general term' below) among the basic terms and render the approach rather uninteresting, or (ii) we cannot infer all entities. ${ }^{20}$

Let me elaborate on this. As the solution to the qua problem suggests, we need categorial terms to ground reference where Thomasson calls terms 'categorial' if they are "highly general sortal terms" (2007, p. 41). But how did we get the categorial terms necessary to get to successful reference grounding? One possibility is to assume them to be basic. But whatever sense of 'basic' this is, it does not match the 'basic' in the description of 'basic term'. For, as the examples Thomasson (2015, p. 106) brings forward also show, these are highly specific terms such as 'cup' or 'teddy'. Thomasson suggests that we can arrange terms with respect to their "specificity" (2007, p. 41). These orderings lead to "hierarchies of increasing generality" (2007, p. 41). If we start an ordering with highly specific terms, then we can take basic terms to be near the very bottom. The grounding of reference of a term $T$ is accomplished by using a less specific, and, therefore, more general, term $T^{\prime}$ and placing some condition on it; for example, I used the more general term 'animal' in order to specify the term 'human being' in the above example. But the idea of starting with basic terms suggests that

\footnotetext{
20 See my (2020: §3). Cf. also the related, but somewhat different dilemma posed by Contessa (2016: 766ff.) which is based on whether or not easy arguments are 'ampliative inferences'.
} 
we have to start with quite specific terms. Thus, we need to use these basic terms in order to introduce less specific terms.

Note, though, that even the hierarchies of generality have a limit: terms such as 'individual', 'object', or 'thing' are not included, because they "are not associated with the application and coapplication conditions needed to provide disambiguation" (2007, p. 42). This claim is central in many of her arguments against other positions: Thomasson regularly points to the use of 'thing' and 'object' in such views and dismisses the positions on this very ground [for a recent example, see her (2017, p. 773)].

However, the idea of using the basic terms to introduce all other terms leads to the following problem. Again, grounding of reference works by taking a categorial term and imposing certain conditions on it. ${ }^{21}$ Hence the most general term we are able to introduce in this manner is the categorial term itself (for example, if the condition is vacuously satisfied). This means that we cannot introduce anything more general than the most general term we have at our disposal. ${ }^{22}$ Therefore, the above characterization of 'basic' — see Thomasson $(2015$, pp. 104, 106)—blocks the successful introduction of anything more general than such basic terms. ${ }^{23}$ Thus, if we want to be in a position to introduce something as general as the term 'number', we have to assume a term at least as general as such a term as 'number' among our basic terms.

Let me illustrate this by considering the foundations of mathematics as provided by ZFC set theory. As this is merely an illustration of the more general argument and points from above, we can ignore other ways of providing set theoretic foundations. Moreover, we need to recognize that the illustration and the illustrated are not a perfect match so that we have to be specific of which parts correspond to which and what's just an artefact of the illustration. In particular, we are not too interested in the exact formal details or all the different ways of providing such, and we don't intend to suggest that every feature of the illustration generalizes. In particular, the following illustration is not meant as arguing for or endorsing a particular position within the philosophy of mathematics.

ZFC set theory is commonly used as foundation of mathematics because we can define all concepts contemporary mathematics needs in terms of sets. ${ }^{24}$ However, the language of set theory is a first-order language with a primitive membership-relation

\footnotetext{
21 In terms of definition, this aligns reference grounding with definitions of the genus-and-differentia form; see Hempel (1952, p. 5f.) and Suppes (1957: §8.1). See also Thomasson’s (2007, p. 41f.) own example.

22 If we allow for the introduction of new terms via disjunction, then the most general term is a disjunction of the terms at our disposal. However, there is the danger that building the disjunction of many such terms puts us into a position in which we can even introduce 'thing', 'object', or 'individual' — which are not part of any of the hierarchies (Thomasson 2007, p. 42).

23 Again, if we allow for the introduction of new terms via disjunction, we get to a term that is the disjunction of the basic terms. This, however, does not suffice to introduce something like 'number' if there wasn't already some rather general and abstract term available. Also, the disjunction might be infinitely long and so not even be a proper part of the language.

24 Of course, set theory is also considered a foundation of mathematics because of its ontology, i.e., of its (broadly Quinean) ontological commitments. Not only can we introduce the necessary definitions, but we have enough entities to answer to them if we take the ontological commitments at face value. This leaves it, of course, open to be a nominalist with respect to such entities and take, for example, the mathematical ontology to be part of a fiction (cf. Field 1980 and Yablo 1998).
} 
$\epsilon^{25}$ whereas the ontology of set theory consists solely of sets. In the language of set theory, we can define 'set' by (something like) 'set $(x): \leftrightarrow \exists y(x=y)$ ' since ZFC only talks about sets so that everything is a set. Of course, as just noted above, this is not a way of providing application conditions for 'set', i.e., such a definition is an artefact of the illustration. We can, of course, use ' $\in$ ' to define 'set', but for the sake of the illustration, I ignore this option. The reason to ignore it is simply that the problem illustrated would not disappear, but simply be pushed back to be about $\in$; however, I take it that it is more natural in our context to work with 'set' instead of ' $\in$ ' because 'set' is a sortal and so in line with the main target of (E).

This brings us to the illustration. Let us illustrate application conditions with definitions and basic terms (in the sense of Easy Ontology) with primitive ones (as understood in definitions). We could do with just the one primitive term 'set' (illustrating the case $n=1$ ), or consider a larger base and take 'set' and 'function' to be primitive terms (illustrating the case $n=2$ ). Again, 'set' might be understood in terms of ' $\in$ ' (which we also allow ourselves to use here), but for the sake of illustration this is irrelevant. Now, given the two primitive terms, the quest is to provide them with definitions. This is easy enough in the case of 'function': as usually understood in ZFC, every function is a univalent relation and every $m$-ary relation can be identified with a set of ordered m-tuples; these, in turn, can be identified with certain sets such as in the Kuratowski-Wiener definition. In this manner, we get a non-circular specification of the term 'function': every set of this-and-that form (i.e., read the analysis provided in the previous sentence backwards) is a function.

However, as 'set' is our solely remaining primitive term, we are in no position to provide it with a non-circular definition. ${ }^{26}$ This situation illustrates also why axioms of theories are often thought of as implicit definitions ${ }^{27}$ : the reason is simply that we cannot non-circularly define all terms. Relating this back to Easy Ontology, the insight is that we cannot provide non-circular application conditions for all terms.

Similarly for any other competitor. Consider, for example, category theory to be the proper foundation of mathematics. It relies on the notion of 'arrow' that cannot itself be non-circularly specified. The problem is simply that we cannot give a proper definition of our basic terms with the resources at hand. We can, of course, go to another theory and provide such definitions. But this is only a way out if we can specify all the terms of this theory. (This is essentially the objection discussed in Sect. 4.2.) Now, as condition (AC1) makes obvious, the application conditions of (basic) terms need not be definitions. Thus, partial definitions suffice to satisfy this condition. But, nevertheless, we also do not have the means to give any kind of application conditions in a language that rests solely on such terms because we do not have any other terms left which could specify them. The example of the implicit definitions shows exactly this: we have to specify (the behaviour of) basic terms circularly.

\footnotetext{
25 Thanks to an anonymous referee to pressing this point and the following.

26 Again, we might use ' $\in$ ' to provide it with such, but then the problem is to provide ' $\in$ ' with appropriate non-circular application conditions; and as we use it to introduce 'set', we cannot use 'set' in its application conditions.

27 Cf. Hempel (1952, n. 26, p. 81), Horwich (1998: ch. 6), and Hale and Wright (2001: ch. 5); for an overview, see Gupta (2019: §2.5).
} 
This is also unproblematic in the case of mathematics because-if we, for example, subscribe to Realism about mathematics-we only want to describe an existing ontology. ${ }^{28}$ It might not even be problematic for other metaontologies. But it is problematic for Easy Ontology — and this for at least two reasons: first, Thomasson imposes a non-circularity condition (AC2) and, thus, exposes her account to such an objection. Second, as we will see in Sects. 4 and 5, just giving up this crucial condition (AC2) is even more damaging for her account than can, prima facie, be expected. This shows that it is not particularly easy to 'take it easy' as the title of Thomasson's (2017) suggests. $^{29}$

\section{Potential objections to the circularity charge}

I mainly want to discuss two objections to the above argument. The first, discussed in Sect. 4.1, draws on condition (AC1) which states that application conditions "needn't be statable" (Thomasson 2015, p. 91). The second objection, discussed in Sect. 4.2, is that we might be able to supply non-circular application conditions for all basic terms if we invoke another language. Section 4.3, then, briefly discusses some more possible objections such as the possibility of an infinite regress.

Before discussing the objections, let me informally point out the general dialectic of my arguments. We have seen in Sect. 3 that there is a circularity problem. The following objections try to show either that such a circularity does not, in fact, arise, or that the circularity is not problematic. In order to do so, I will consider several strategies-be they Thomasson's or not. I will show then the following: either the strategy is unsuccessful, or Easy Ontology loses its motivation. I take it to be clear that both options are problematic for Easy Ontology.

\subsection{Objection 1: unstatability}

Recall condition (AC1) from Sect. 2.2; it states, among other things, that application conditions are "semantic rules of use" which "needn't take the form of necessary and sufficient conditions, and needn't be statable" (2015, p. 91). The objection to the above circularity argument is effectively to point out that all that the argument shows is that the application conditions of (some) basic terms are not statable. But as this was never presupposed, Easy Ontology is not threatened by my argument.

Before answering it, let me first point out that this objection is implausible as Thomasson herself wants to supply application conditions. She claims that

we can show that one may answer an existence question 'Do $K$ s exist?' easily, by appeal to whether the application conditions for ' $K$ ' are fulfilled, if we can show a way to state and understand the application conditions for ' $K$ ' that does

\footnotetext{
28 On this issue, cf. Gödel's (1944, p. 455ff.) discussion of impredicative definitions; see also Gupta (2019: $\S 2.6)$.

29 See also Button (2020: §2.4) for an argument that “"easy” ontology is something of a misnomer” (2020, p. 41). His specific reasons are different from the ones provided here, though.

Note, again, that this is the crucial difference between this paper and Brenner's (2018).
} 
not appeal to $K \mathrm{~s}$. A little ingenuity enables us to see how application conditions even for these (relatively) basic nouns of the thing language could be stated in terms that don't appeal to the existence of those very things. $(2015$, p. 106, my emphases)

Indeed, her specific proposal is to specify the application conditions as we discussed them in Sect. 3.2, viz., the application conditions for a basic term like 'cup' are provided by 'cupwise arrangement of particles'. Thus, she seems to feel the need herself to state application conditions for basic terms. Of course, we could just take that to be a slip and disregard it. Instead, an Easy Ontologist can insist on the non-statability of some of the application conditions. So let us consider this route.

Before doing so, let me also point out again which particular problem needs to be resolved-if only in order to see what would count as legitimate objection to my argument. As I put it in Sect. 3.2, the problem concerns reference grounding. Recall that the particular motivation for application conditions is that they (allegedly) resolve the qua problem (Sect. 2.2). What my argument establishes is, effectively, that Easy Ontology still has such a problem at its hand. For, we need the basic terms to disambiguate, i.e., resolve the qua problem, for derivative terms. But this can only then be successful, if the basic terms are not themselves riddled with such a problem. The specific way proposed to circumvent this problem is to supply application conditions. But, as we have seen, the application conditions of (some) basic terms are either non-existent or circular - and both options are not apt to resolve any ambiguity. It is important to bear that in mind when we discuss (Thomasson's) strategies to supply application conditions to such basic terms. ${ }^{30}$

Furthermore, I flagged already in Sect. 2.2 that the phrase 'needn't be statable' is ambiguous. Indeed, both the 'needn't' and the 'statable' are somewhat unclear. I will go through several possible readings of them; however, as there might be more possible cases than anyone can consider, I will rather pose a challenge to the Easy Ontologist and indicate why I think it cannot be met.

Let us start to distinguish different ways in which something is not statable. One way is that, so far, no one has come up with a way to state the application conditions, but it is perfectly possible to do so. This, however, cannot be the relevant sense as the above argument certainly applies to it.

Another relevant sense-and, indeed, the one suggested by Thomasson (2015, p. 91ff.) - is exactly the above one. She draws an analogy with grammatical rules when she explicitly states that application conditions "should be thought of as semantic rules analogous to grammatical rules" (2015, p. 92, my emphasis). Even though many of us cannot state the grammatical rules, we have mastered them and count as competent speakers. This sense of 'unstatable' is certainly uncontentious-but it is also irrelevant here. Indeed, even though most of us cannot state the grammatical rules, there are experts who can. Also, we could acquire this knowledge rather easily by picking up the right book. Thus, this case is covered by the above circularity observation. My argument applies to the case of experts; and this cannot be dismissed on the basis that

\footnotetext{
30 Thanks to an anonymous referee to pushing me on several of Thomasson's strategies to prevent a circularity as well as on insisting that application conditions might be unstatable. This led to several changes in this as well as in the following sections.
} 
ordinary people are not experts. Besides providing a disambiguation for 'unstatable', this also provides us with a reading of 'need'. It is true that we don't need to state grammatical rules explicitly in order for our sentences to be grammatical; nevertheless, such rules exist and there are people who can explicitly state them. Indeed, they even are explicitly stated in grammars.

Another suggestion for an appropriate sense of 'unstatable' is the following: Application conditions of certain terms are properly unstatable by anyone. This, too, is problematic for Thomasson. We'll come to this case shortly after considering intermediate cases.

Another way for the application conditions to be unstatable is for them to be too long to actually state them, i.e., we would need a language that allows for infinitely long sentences. This case, too, is covered by the above argument. Even though the conditions (e.g., the ' $\psi\left(T_{1}, \ldots, T_{n-1}\right)$ ' from Sect. 3.2) are then infinitely long, the circularity charge would still apply. (We'll come back to such a case below.)

A related case would be the one in which we allow for uncountably many distinct (basic) terms. In such a case a countable language could not distinguish between all of them with its resources. Thus, some of the terms would be unstatable in the countable language. And this would be a case in which they really are unstatable. Compare, for example, the case of a first-order language. It is well known that some sentences are not 'firstorderizable', i.e., there are sentences which cannot be represented by means of a first-order formula (see, e.g., Boolos 1984a, b); one such sentence is the well-known Geach-Kaplan sentence "Some critics admire only one another". Another example of a property that cannot be expressed within a first-order setting is finiteness.

If this is the relevant sense of 'unstatable', then it does not help the Easy Ontologist. For one thing, the problem arises in well-defined and fixed languages such that a simple change of language circumvents the problem (changing from first-order to second-order or from singular first-order to plural logic). Also, Thomasson is keen to emphasize the fact that she is using ordinary English, ${ }^{31}$ i.e., a natural language, and natural languages might not have the same problems as their formal counterparts. Indeed, intuitively speaking, ordinary English is capable of expressing finiteness as well as sentences such as the Geach-Kaplan sentence-as I just did above. ${ }^{32}$

For another, and this brings us back to the above thread, if ordinary English is indeed not capable of expressing some of the application conditions, rendering them unstatable, then we cannot answer the corresponding existence question. For, as we have a name of the (kind of) entity, i.e., a term, we can ask whether this term (E)applies. But, as the application conditions tell us what to look for, we are in no position to answer such an existence question-at least not by easy means; there might still be a hard way.

\footnotetext{
31 See Sect. 4.2 for the relevant references.

32 Thanks to an anonymous referee for pointing out that there might be a problem with respect to what counts as expressing a concept. I take it that, intuitively, using the corresponding term suffices here. Nothing much hangs on it for myself. However, an Easy Ontologist is invited to challenge this. The challenge is, then, to provide an account of adequately expressing something that does not conflict with application of principle (E) as well as with its claim that Easy Ontology concerns natural language. In any case, my following point is not affected by this.
} 
The objector might argue at this point that Easy Ontology only claims to answer well-formed existence questions and this would not constitute one. But, to be a wellformed existence question, the respective term must have application conditions [see the discussion of (AC1) in Sect. 2.2 above as well as, e.g., (2009b, p. 449 and 2015, p. 129)] — and, by assumption, it does; they just are not statable. Therefore, such an existence question is in the range of questions that Easy Ontology claims to give an answer to, or, as Thomasson puts it, "all well-formed existence questions may be answered by conceptual and/or empirical work" (2015, p. 128). But as the conceptual work is bound to the application conditions, this option drops out. So, it is the burden of the Easy Ontologist to provide reasons that terms with unstatable application conditions are such that they are empirically accessible.

But here is a problem in providing such reasons. The specific motivation to introduce application conditions is to circumvent the qua problem. The qua problem is a problem of the indeterminacy of reference. So, if the application conditions of a term $T$ are unstatable, then we can likewise never have reasons that we even established reference to the entities in question. This, then, blocks empirical inquiry until we have reasons to believe that we are actually inquiring about $T \mathrm{~s}$. And any relevant reason will give us a way to (at least partially) state application conditions for the term, rendering its application conditions statable after all—and exposing such a term to the circularity charge. ${ }^{33}$

Thomasson's strategy to circumvent the problem of stating application conditions in order to answer existence questions is to suggest that

it is plausible that any language must include some 'semantic basic term', that is, terms that cannot be learned just by way of learning definitions stated in other terms. (2015, p. 92)

And she continues:

And if that is so, we must allow that for at least some terms, the application conditions (considered as semantic rules of use) needn't be capable of being (informatively) stated to be learned and to be in force. Instead, speakers may learn to master the rules of use for those terms by other means, for example, ostensively as we learn that a term is to be applied in situations like this (and not in situations like that), or via judgments of similarity to ostended paradigms[.] (2015, p. 92, her emphases)

Thus, these 'semantic basic terms' are supposedly learnable via ostension and similarities among the referents of such ostensions. Furthermore, when teaching a new term, we "simply demonstratively [apply the term] in some situations and [refuse] it in others, and [applaud] or [correct]" (2015, p. 93) the attempts of the child.

Nevertheless, as all the other terms are built on basic ones, we run into the problem I pointed out above. If we are not in a position to fix the reference, i.e., to circumvent the qua problem, then—according to Easy Ontology—we cannot even evaluate existence claims. I already flagged the issue in Sect. 2.2, viz., changing to ostension only helps

\footnotetext{
33 As already pointed out in footnote 19, any other term used to get us closer to the application conditions has to be properly introduced-but, given Thomasson's (2015: ch. 8.2) account of introducing new terms to a language (see also my 2020), this is impossible, if the basic terms themselves are already problematic.
} 
if there is not an analogous qua problem-but there is. It is indeterminate to what someone is pointing.

There are at least two problems with Thomasson's suggestion. The first has to do with indeterminacy and the second with the relevance of this account. Let me consider these in turn.

First of all, it is hard to imagine a way in which you teach someone the use of a term by telling her/him that it applies 'in situations like this' if s/he has not already mastered a good amount of language; but then such a term can hardly be semantically basic. It is difficult enough just to point to something to make someone else understand what is referred to; and pointing to a situation makes the task rather more difficult. When does the situation start? When does it end? How shall we distinguish the pointing to a situation as distinct from the pointing to an object $?^{34}$ And since the similarity is with respect to 'ostended paradigms', it simply inherits the problems I just presented. By and large, this way of interpreting the unstatability is rather not available to Thomasson as she encounters problems she takes to be serious herself; indeed, they are the very motivation to introduce application conditions in the first place. Thus, if she does not recognize a qua problem here, then she loses her own motivation to introduce application conditions because they are not needed to disambiguate reference. ${ }^{35}$

This brings us to the second problem. The problem is that Thomasson's account presupposes someone who has already learned the language and now teaches it to someone else-but this means that we must be in a situation in which the problem my argument gets at is already solved. Thus, even if this is how we learn language, the particular issue is not addressed. As I put it at the beginning of this section, the problem is reference grounding. Teaching children language presupposes that this problem is already solved. The children can only pick up the correct use of terms from people who use the term correctly. As we have seen, this presupposes that the term has application conditions (whether or not they are statable). Thomasson (2007, p. 48) subscribes to a 'hybrid' causal theory of reference. The application conditions are necessary to ground reference-and this is the non-causal part-in order for the reference of the terms to be then passed along causal chains. The teaching of terms corresponds to the causal part of this chain-but the problem is with the grounding of reference. Thus, the specific way in which children are taught particular terms is irrelevant for the discussion here. On the contrary, this aspect rather shows that something is wrong with Easy Ontology. If reference grounding works in this particular way, we lose, again, all the motivation to introduce application conditions in the first place.

To sum up, any understanding of 'unstatable' faces such problems. Thus, the burden is on Thomasson to provide a reading of 'needn't be statable' that does not invite criticisms along the above lines. I fail to see how she can provide such a reading that does not undermine her own approach.

\footnotetext{
34 Cf. Quine's (1960: ch. 2, esp. §12, p. 52f.) 'Gavagai' argument in support of his 'inscrutability of reference' thesis; see also his (1960: $\$ 21$, p. 100ff. in ch. 3) as well as Putnam (1980, p. 17). Note, however, that I only rely on the indeterminacy of ostension, and don't invoke an indeterminacy of translation or inscrutability of reference thesis.

35 This does not imply that I am objecting to us learning language in some such way. The point is simply that going this way undermines the motivation for Easy Ontology.
} 
Lastly, there does not seem to be a reason why the application conditions should be unstatable at all. To follow up on the 'grammatical rules' analogy, there is no problem in providing a comprehensive grammar. ${ }^{36}$ There is, of course, a problem for Thomasson's account. But the lesson to be learned here can rather be: we do not have any reason to believe that (basic) terms even have application conditions. And, indeed, as the argument of Sect. 3.2 shows, if they have them, they are circular. As shown, either way is damaging for the easy approach.

\subsection{Objection 2: change of language}

This brings us to the second objection which is related to the idea of theory change (see the end of Sect. 3.2). The first longer quotation in Sect. 4.1 might be understood as a change in language, i.e., 'particles arrange cupwise', for example, is a term of another language that provides application conditions for 'cup' in our language (or, as in the quotation, Carnap's 'thing language'). As there is a change in language, the circularity is prevented.

I will first consider two replies to this objection. The first is to try and play the suggestion out in order to see in what way it is problematic; this will also be helpful for our discussion in Sect. 5 as it involves analytic entailments. The second reply is to point out that we just run into another circularity problem. After these replies, I will consider another of Thomasson's strategies to get around the circularity, viz., to use a 'feature-placing language' in order to supply application conditions.

The first way to see that this objection is a non-starter is to note that Thomasson is very keen to emphasize that she is talking about and using ordinary English (see, e.g., 2007, pp. 33, 35, 2008, p. 72, 2015, pp. 25, 41, 44, 176, 2016, p. 125f., 2017, pp. 769, 771 ) and it is ordinary English that makes the inferences obvious (2017, p. 769), i.e., easy. So it would be rather surprising to have a change in language here.

In particular, many (if not all) of the easy arguments are either not easy or not valid anymore if we have to change the language in-between. For, we want to establish the existence of entities by using principle (E). But this principle is supposed to be a principle of ordinary English. Since some steps in the easy arguments would involve a change of language, principle (E) is not applicable anymore.

On the other hand, if we assume principle (E) still to be applicable, we need an explanation for the alleged obviousness of the arguments. If one step of the argument builds a bridge to another language, this cannot be obvious to anyone who has not mastered both languages. Further, even if all the steps of such arguments are justified, the steps are not easy as there is no reason that a valid argument in another language involves only easy steps.

Moreover, the obviousness and easiness of the arguments stems from analytic entailments. Again, these analytic entailments are part of ordinary English. Therefore, if we interpret easy arguments as involving a change of language, then at least one step of such an argument cannot rest on analytic entailments in this sense.

\footnotetext{
36 Indeed, if we take the analogy seriously, the suggestion is: there are certain bits of language which don't have any grammatical rules. On the face of it, this is just implausible. This shows that the analogy is not particularly well-suited for the case at hand.
} 
We can also note here that analyticity is a relation on sentences of one language and not on sentences of different languages - as Thomasson (2015, p. 242, n. 10) points out herself. It is maybe a plausible suggestion that there are languages which are related in such a way that sentences of these languages stand in such a relation, too. However, that presumably makes only sense if the one language contains all the sentences of the other language so that this sense of 'analytic' is analogical to the analyticity within one language.

However, even if we allow for such a relationship for sentences of different languages, say English and German, easy arguments can still only then count as easy if both languages are mastered. And even in such cases it is unclear whether the analytic relationships of German sentences match up the analytic relationships of English sentences, even if we have a German sentence $G$ and an English sentence $S$ such that it can plausibly uphold that $S$ analytically entails $G$ or vice versa. Another German sentence $G^{\prime}$ might be analytically entailed by $G$ without being analytically entailed by $S$. But if we pass from $S$ to $G$ and from $G$ to $G^{\prime}$, it is not clear whether we end up with the required English sentence $S^{\prime}$ that puts us in a position to use (E) and infer the existence of some entity.

Let us suppose, however, that even the change-of-language step can be considered to be obvious and easy. Demanding the steps in arguments (except the step involving a change of language) to be easy, we need a guarantee that the steps in the other language are easy, too. But there is no such guarantee forthcoming since the other language does not need to align in the right way to match up analytic entailments in ordinary English [see, e.g., Hirsch's (1993) for examples of languages that are able to express the same things but without this strong analytic connection]. This shows that this strategy fails, too.

Another way to see that this strategy fails is as follows. The change-of-languagestrategy can only then be taken to be successful, if we don't end up in another circle, i.e., if we don't end up in a situation as dismissed in Sect. 3.1. What we effectively do when supplying application conditions for the basic terms of English by recurrence to German is to enrich our pool of basic terms. However, the circularity pointed out in Sect. 3.2 is not directly circumvented in this way. Rather, we push the problem one step back and have to ask, in the running example, what the application conditions of the German terms are. As we cannot use the English terms, we run into a circularity problem regarding the German terms. And this problem repeats itself if we try to supply the application conditions of German terms by means of yet another language.

The problem, as we have just seen, is that the languages we used in order to supply application conditions rely themselves on terms. However, Thomasson also considers another type of language:

Or one could perhaps instead express the application conditions in [...] a 'featureplacing language', and hold that if it is cupping around here, then there is a cup. If so, we could state the application conditions for 'cup' without appealing the existence of a cup or indeed of any object at all. (2015, p. 107)

The idea is that we use a language that does not appeal to any particular object in order to supply application conditions for the corresponding terms. This amounts to saying that the application conditions of a term $T$ do not involve any other term $T^{\prime}$ so that 
the circularity is prevented, viz., terms correspond to objects, but 'it is cupping around here' does not correspond to an object. (The alternative is that 'it is cupping around here' does correspond to an object; but then there is a term $T^{\prime}$ and the circularity is not circumvented.)

Brenner (2018, p. 610f.) already considers and rejects this strategy, and I agree with his assessment. According to him, we have two options of interpreting sentences like 'it is cupping around here', viz., either such sentences have ontological implications or they don't. In the latter case, we run into a problem as they are supposed to imply sentences of the form 'there exists a ...' which are, in turn, ontologically loaded according to principle (E). This means that we have to understand such sentences as having ontological implications, but then they are not helpful for the case at hand as they are just different formulations of sentences of an object-placing language.

Adding to this, we can also note that we potentially run into the problem of grounding the reference. The application conditions are supposed to ground reference. As Thomasson presented the case, this is accomplished by invoking categorial terms. If the feature-placing language does not have those, it is either not capable of grounding the reference of terms like 'cup', or it cannot come with its own qua problem. But in the latter case we lose all motivation to introduce application conditions again. Thus, either we don't solve the motivating problem to introduce application conditions, or we lose the motivation to introduce application conditions. Either case is obviously problematic.

\subsection{Further objections}

In this section, I want to consider further objections. As Brenner (2018) already discusses these issues, I will be quite brief.

One way of circumventing the circularity charge is to endorse an infinite regress. This is also a possible way out if we try to use another language to supply application conditions of the terms in the language we start with (see Sect. 4.2). In Sect. 3.2, I did not mention the possibility of an infinite regress because Thomasson's strategy to divide terms into basic and derivative ones together with her characterization of 'basic' seems to suggest that we start from a finite base of basic terms which are used to introduce all other terms. However, as my argument shows, we could easily go for an infinite regress as well. Suppose that we have infinitely many (basic) terms $T_{1}, T_{2}$, $\ldots, T_{n}, \ldots$, such that

$$
A C\left(T_{1}\right)=\varphi_{1}\left(T_{2}\right) ; \ldots ; A C\left(T_{n}\right)=\varphi_{n}\left(T_{n+1}\right) ; \ldots,
$$

i.e., the application conditions of a term $T_{i}$ are provided in terms of (at least) a term $T_{i+1}$.

Note, first, that if we even allow for uncountably many basic terms, we end up in a situation that has already been covered by the discussion of 'unstatable' in Sect. 4.1. So, let us assume that this regress is meant to introduce countably many terms. Likewise, we can assume that we do not just have infinitely long sentences invoking many basic terms in a circular fashion because, otherwise, we end up in another circle, 
as also discussed above. Thus, for every basic term $T_{j}(j \in \mathbb{N})$, either $A C\left(T_{j}\right)=$ $\varphi_{j}\left(T_{j_{1}}, \ldots, T_{j_{n}}\right)(n \in \mathbb{N})$, i.e., the application conditions of $T_{j}$ involve finitely many other basic terms (that have not already been used, i.e., $j_{i}>j, 1 \leq i \leq n$ ), or the application conditions are themselves infinitely long.

Essentially, both options are open to Brenner's observation that because Thomasson describes the application conditions of terms as meaning-constitutive, it "seems implausible that we could learn an infinite sequence of sortals, and their terms' associated application conditions" (2018, p. 608). We can even make this charge more pressing by recalling Thomasson's characterization of 'basic term' as terms "we tend to learn early in our cognitive or linguistic development" (2015, p. 104)—but even allowing the possibility that we have infinitely many terms at our disposal, we certainly do not learn infinitely many terms in our early cognitive and linguistic development. Thus, Thomasson's only option here is to suggest that basic terms are "innate 'input analysers' [which] give us the conceptual rules which we need [and] only link with the relevant term of our language" (2015, p. 93). In the case of the regress, this means that we must have infinitely many of such innate terms which seems implausible (cf. Brenner 2018, p. 608).

Further, Thomasson suggests the following:

And in fact, we teach our children words not by telling them (unhelpfully) that they should apply 'dog' wherever a dog exists, but rather by simply demonstratively applying 'dog' in some situations and refusing it in others, and applauding or correcting their attempted uses of phrases like '[there is a] dog' in various situations. (2015, p. 93)

The problem is, however, that we end up in a situation as above. By the lights of the Easy Ontologist, we have a qua problem here. It would not be enough to just say 'dog' without disambiguation. This would not be a problem per se, but it is for the case at hand, viz., for 'semantic basic terms', i.e., the very first words we learn. Whatever reason there is for children to pick up the correct use of words, it does not seem that Easy Ontology fits the picture with its need to supply application conditions. Indeed, it rather seems that Thomasson supplies a picture of language acquisition that is contrary to what she claims in other contexts.

It might be objected here that there are two different things going on. The one is language acquisition, the other one disambiguating reference. Thus, the issue of language acquisition should be kept separate form the latter-and it is only to the latter for which a qua problem arises. Clearly, to teach children language already presupposes that there is someone who has acquired the language properly; who else would teach the children? However, if the Easy Ontologist plays this game, it is still difficult to see how we ever got to this point that someone acquired a language in the first place. In particular, the semantic basic terms of such a person may very well differ from the semantic basic ones of anyone s/he teaches her/his language to. But all the ontological questions and the easy game should be applicable to such a person-and this is where we run into the circularity problem again. As discussed in Sect. 4.1, teaching language to children presupposes that we have resolved the problem of reference grounding - but, as I argued, this problem is wide open. 
Note, too, that it is implausible to suppose that whoever managed to first fix the reference of a term had 'innate input analysers' in the sense needed to make Thomasson's suggestion work. For, such a person would do the first steps in even getting to the concepts which then might become innate in her/his offspring. Even today we introduce new concepts to our available stock and this does not seem to answer to anything 'innate' at all. It might be objected that we have enough concepts around to ground reference to a new concept. However, this means again that we are only getting more fine-grained with respect to what we are able to introduce because we'd need a grounded concept that gives us at least a rough idea what we are introducing. In this sense, we would not be able to properly introduce anything really new, but only to be more and more specific with respect to what we refer; otherwise we'd need categorial terms which, by assumption, we don't have for these new terms. Thus, this option, too, is not doing the work that the Easy Ontologist requires it to do. (Consider the case of 'electron'. Presumably we are now in a position to disambiguate the reference by invoking terms such as 'matter' - terms on a par with 'object' and 'thing' that Thomasson rejects.)

With respect to the circularity charge, Brenner (2018, p. 609) discusses two reasons why the circularity is bad. The first reason is that Thomasson explicitly invokes her noncircularity condition (AC2), the second that circular application conditions "make no substantive requirements on the world for their satisfaction" (2018, p. 609). I covered the first one already above in Sect. 3.2, so let me just say something about the second. In the way I set up the argument, this concern is covered as well as I don't think my argument relies on terms with non-trivial application conditions, but also applies to the latter. Thus, I don't need another reason for the non-substantive terms to be problematic.

\section{How damaging is the circularity for easy ontology?}

Having refuted the objections, let us consider which impact the circularity charge has on Easy Ontology. The main ideas have already been invoked in Sects. 3 and 4; here we consider how widespread they are. Before doing so, let us consider how Thomasson herself thinks about her Easy Ontology and the potential impact of a circularity in the approach. She wants to downplay such a circularity; I, on the other hand, argue in this section that the circularity is more damaging than she makes it appear to be.

Thomasson characterizes any approach as 'easy' if it has "two features" (2015, p. 128):

EF1 "all well-formed existence questions may be answered by conceptual and/or empirical work" (2015, p. 128), and

EF2 "at least some disputed existence questions may be answered by means of trivial inferences from uncontroversial premises" (2015, p. 128).

We consider the impact on these features; I argue that neither is satisfied if Easy Ontology is circular. 
Before giving her argument that the non-circularity condition (AC2) can be fulfilled, Thomasson also tells us what's at stake if she is not able to make her case for the basic terms. Let me give the quotation in full:

Indeed, one might worry that the application conditions for basic sortal terms ' $K$ ' must appeal to the existence of $K$ s (rather than their being fulfilled by the existence of entities of other sorts) - making it impossible to appeal to whether or not application conditions are fulfilled to determine whether things of the relevant sort exist, and so rendering the easy approach to existence questions unworkable for this range of terms. Of course it might still provide an easy way of resolving existence debates for many disputed entities, including numbers, properties, propositions, events, states, nations, and so on, and so still might be quite a significant contribution to metaontology. But, the objection goes, hard ontology on this view still has a place: existence questions posed using basic sortals must be addressed using hard ontological methods, and the rest may be answered easily. (2015, p. 103f., her emphasis)

So, Thomasson reasons that even if she can't make the case for principle (E) applying in the case of (some) basic terms, she still has a lot going for cases in which the terms are derivative such as, for example, 'number' (2015, p. 104). ${ }^{37}$ Thus, Thomasson makes it sound as if nothing much is at stake here, and that the easy approach stands its ground still for a large class of entities. I beg to differ.

First, given her own views, it is rather unclear how the basic terms that have to be invoked in the application conditions of derivative ones do circumvent the qua problem. For, according to Thomasson's own reasoning, we cannot successfully refer to anything without using a categorial term that helps to ground the reference. To this end, she introduces the idea of application conditions. But, so far, she was not able to make plausible that those basic terms even have application conditions. Indeed, the argument of Sect. 3.2 shows that they can only have circular ones. Granting them such, however, leaves us with two choices given (EF1). One is to admit that we do not have a way of conceptually determining whether the corresponding entities exist; the other is to do empirical research. But, we already pointed out in Sect. 4.1 that without fixed reference, empirical investigations are doomed to fail, too. As these are the only available options according to (EF1), we end up in a position that still refutes (EF1). For, the terms have application conditions, i.e., the corresponding existence questions are well-formed. Yet, they are not easily answerable. Easy Ontology is not easy.

So far, this just seems to be a rehearsal of what Thomasson already suggested in the above quotation. But things get even worse as soon as we consider how derivative terms are introduced. They are introduced "on the basis of" (2015, p. 99) other terms. Thus, working our way back as far as possible, they are introduced on the basis of basic terms. Indeed, Thomasson makes this explicit when she addresses a 'bad company' problem for her account; she gives "conditions for new sortal terms to be minimally introduced to the unextended language L" (2015, p. 263); the first condition is as follows.

\footnotetext{
37 Interestingly, Thomasson sees her account as not endangered for terms which do not have 'non-trivial application conditions'. This shows that Thomasson would not be particularly worried about Brenner's (2018) arguments.
} 
The term(s) must be introduced via a conditional that gives sufficient conditions for its(/their) application, stated using the extant terms of $L$ and/or other minimally introduced terms. (Note that this permits that we may simultaneously introduce interrelated terms [...]. But it requires that to do so there must be sufficient conditions for all of them statable in terms of L or other minimally introduced terms.) (2015, p. 263, my emphases)

Thus, we are only allowed to use terms that have been 'minimally introduced' ${ }^{38}$ As we have to start with a certain set of terms, viz., the basic terms, every other term must be statable in terms of the basic terms we started with. For, otherwise, we would end up with terms that have not been 'minimally introduced', contrary to what the quotation enforces.

As argued in Sect. 3.2, some basic terms have circular application conditions, i.e., if $T$ is such a basic term, its application conditions are " $T$ " applies iff $T$ s exist'. But, as argued, any other (basic) term without circular application conditions must invoke among its application conditions circular terms. Thus, we can count such terms as derivative in the relevant sense here.

Furthermore, as the existence of some entity is, by (EF2), easily inferable from the existence of other entities, we need corroboration of the existence of the latter. Put differently, easy arguments work via analytic entailments, i.e., we start from "uncontroversial premises" (2015, p. 129) and apply such entailments to derive the existence of something else - and the application of the latter must be statable in terms of the basic terms we started with (as per the above quotation).

If we really do the ground work here, the uncontroversial premise must involve basic terms. We can note that, by the observation in the above paragraphs, the premise is presumably not uncontroversial after all-as the term needs proper application conditions to guarantee successful reference; but we have seen that this condition is not satisfied. Putting this problem to one side, we can still wonder what analytic entailments are possible from application conditions of the form " $T$ " applies iff $T \mathrm{~s}$ exist' (or, 'if something is a $T$, then it is a $T$ '). Prima facie, there seems to be no reason that anything interesting follows analytically. An analytic entailment presumably is only established, when the conditions of the entailed sentence are satisfied in virtue of the satisfaction of the conditions of the entailing sentence. ${ }^{39}$ For example, if the application conditions of a term $T^{\prime}$ are "“ $T^{\prime \prime}$ " applies iff $T_{1}$ s exist' (or, 'if something is a $T_{1}$, it is a $T^{\prime \prime}$ ) and we have a sentence establishing the existence of $T_{1} \wedge T_{2} \mathrm{~s}$, ${ }^{40}$ i.e., a more specific entity, then we can infer that $T^{\prime}$ s exist, too. Likewise, if we have the existence of $T_{3} \mathrm{~s}$ and the application conditions of $T^{\prime \prime}$ are " $T^{\prime \prime}$ " applies iff $T_{3} \vee T_{4} \mathrm{~S}$ exist' (or, 'if something is a $T_{3} \vee T_{4}$, it is a $T^{\prime \prime}$ '), we can infer the existence of $T^{\prime \prime}$ s.

But, again, these are not application conditions of basic terms; the available application conditions don't have that form. In fact, they seem rather to involve atomic sentences, if we take 'exist' to be a predicate; if we take it to be a quantifier, it only

\footnotetext{
38 In terms of theory of definition, Thomasson's condition corresponds to the eliminability of defined terms; see Hempel (1952: §4, p. 17ff.) and Suppes (1957: §8.2).

39 See my (2020: $\$ 2.4)$ for a partial explication of 'analytic entailment'.

40 The ' $T_{1} \wedge T_{2}$ ' is to be understood in the obvious way, i.e., an entity $x$ is a $T_{1} \wedge T_{2}$ iff. $x$ is a $T_{1}$ and $x$ is a $T_{2}$, and similarly in other cases.
} 
follows that something satisfies the formula in question-which we already knew. Also note that even though something satisfies the formula, there is no reason to think that such an existence sentence analytically entails a specific instance; otherwise, it would be a conceptual truth that, given a specific $a$, if there are $T \mathrm{~s}$, then this very $a$ is $T$, which is absurd in most cases (just because there are human beings, it isn't analytically entailed that, for example, I exist). Note also that I am not claiming that no existence sentence has interesting (analytic) implications, but that the specific ones we are considering here, i.e., those involving basic terms with circular application conditions, don't have any. A sentence like ' $\exists x T(x)$ ' might have interesting implications if the ' $T(x)$ ' is complex and involves information about other things. But this is not the case for basic terms. Thus, the only available analytic entailments for basic terms seem to be from something like ' $\exists x T_{1}(x)$ ' to ' $\exists x\left(T_{1} \vee T_{2}\right)(x)$ '.

Moreover, it is even unclear that the application conditions of derivative terms have anything in common with the application conditions of a (circular) basic term. For the application conditions of the basic term $T$ are " $T$ s exist". But the application conditions of the derivative term $T^{\prime}$ are, for example, " " $T$ ' applies". The problem here is that in the one case we are using a term, in the other case we are only mentioning it. Thus, even if we can take it as established that the basic term applies, there is no immediate analytic entailment establishing the existence of anything else. The only entailments that hold are trivial and amount to nothing more than a simple re-naming of the entities corresponding to the terms involved in the entailments, but not to the inference of the existence of (different) entities.

By and large, there don't seem to be (interesting) analytic entailments available (even granting that the concept analyticity makes sense). Thus, if we take away the basis, the complete approach collapses. In particular, if we grant circular application conditions, (EF2) cannot be satisfied, i.e., the approach is-by Thomasson's own characterization-not easy. In particular, then, it seems that Easy Ontology fulfils neither condition (EF1) nor (EF2).

\section{Conclusion}

Let me summarize the results of this paper. In Sect. 2, I provided a sketch of Easy Ontology (Sect. 2.1), motivated Thomasson's reasons to introduce application conditions and provided a partial characterization of them (Sect. 2.2). The main idea of application conditions is to circumvent the qua problem that purely causal theories of reference face. It is Thomasson's self-set goal to give application conditions to terms in a non-circular fashion to make her easy approach work. To this end, she distinguishes between derivative and basic terms.

Section 3 is an argument that Thomasson is not successful in preventing the circularity. In particular, after laying down some preliminaries in Sect. 3.1, I argue in Sect. 3.2 that there must be basic terms which only have circular application conditions. In Sect. 4, I address potential objections to my argument.

Lastly, Sect. 5 evaluates the question of how much impact such a circularity charge has. I argue that it is damaging for Easy Ontology as a whole, even though Thomasson 
claims that the approach might still be applicable to the parts involving derivative terms. Thus, the circularity implies that Easy Ontology is not easy. ${ }^{41}$

Let me emphasize again that my arguments only use what Thomasson herself endorses. This is why there is no reasonable claim that I am begging the question. And as it is Thomasson's strategy to distinguish between derivative and basic terms, this step, too, is not easily dismissed. I conclude then, that Easy Ontology — as it stands - is not a tenable metaontology.

Acknowledgements I would like to thank Julian Dodd, Thomas Schindler, Benedetta Magro, and the audiences of the Open Minds XIII Graduate Conference, of the 11th Arché Graduate Conference (including my respondent Alessandro Rossi), and of the 22nd Oxford Graduate Conference (including my faculty respondent Ofra Magidor) for valuable feedback. I would also like to thank the anonymous referees (and one of them in particular) for their detailed comments and suggestions, and, especially, Jeroen Smid and Chris Daly.

Open Access This article is licensed under a Creative Commons Attribution 4.0 International License, which permits use, sharing, adaptation, distribution and reproduction in any medium or format, as long as you give appropriate credit to the original author(s) and the source, provide a link to the Creative Commons licence, and indicate if changes were made. The images or other third party material in this article are included in the article's Creative Commons licence, unless indicated otherwise in a credit line to the material. If material is not included in the article's Creative Commons licence and your intended use is not permitted by statutory regulation or exceeds the permitted use, you will need to obtain permission directly from the copyright holder. To view a copy of this licence, visit http://creativecommons.org/licenses/by/4.0/.

\section{References}

Benacerraf, P., \& Putnam, H. (Eds.). (1983). Philosophy of mathematics. Selected readings (2nd ed.). Cambridge: Cambridge University Press.

Blatti, S., \& LaPointe, S. (Eds.). (2016). Ontology after Carnap. Oxford: Oxford University Press.

Boolos, G. (1984a). To be is to be a value of a variable (or to be some values of some variables). The Journal of Philosophy, 81(8), 430-449.

Boolos, G. (1984b). Nonfirstorderizability again. Linguistic Inquiry, 15(2), 343.

Brenner, A. (2018). Easy ontology, application conditions and infinite regress. Analysis, 78(4), 605-614.

Button, T. (2020). Deflationary metaphysics and ordinary language. Synthese, 197(1), 33-57.

Carnap, R. (1952). Meaning postulates. Philosophical Studies, 3(5), 65-73.

Chalmers, D. J., Manley, D., \& Wasserman, R. (Eds.). (2009). Metametaphysics. New essays on the foundations of ontology. Oxford: Oxford University Press.

Contessa, G. (2016). It ain't easy: Fictionalism, deflationism, and easy arguments in ontology. Mind, 125(499), 763-773.

Devitt, M., \& Sterelny, K. (1999). Language and reality. An introduction to philosophy of Language. Malden: Blackwell.

Field, H. (1980). Science without numbers. A defense of nominalism. Oxford: Basil Blackwell.

Gödel, K. (1944). Russell's mathematical logic. In: Benacerraf \& Putnam (1983) (pp. 447-469).

Gupta, A. (2019). Definitions. Stanford Encyclopedia of Philosophy (Winter 2019 Edition), Edward N. Zalta (ed.) https://plato.stanford.edu/archives/win2019/entries/definitions/ .

Hale, B., \& Wright, C. (2001). The reasons's proper study: Essays towards a Neo-Fregean philosophy of mathematics. Oxford: Oxford University Press.

Hempel, C. G. (1952). Fundamentals of concept formation in empirical science. Chicago: The University of Chicago Press.

Hirsch, E. (1993). Dividing reality. Oxford: Oxford University Press.

Horwich, P. (1998). Meaning. Oxford: Oxford University Press.

41 And this is the root of the problem not laid bare by Brenner (2018). 
Kripke, S. A. (1972, 80). Naming and necessity, revised and enlarged. Oxford: Blackwell. Originally published in Davidson, Donald, Harman, Gilbert, (Eds.). (1972). Semantics of natural language (pp. 253-355). Dordrecht: Reidel.

Plebani, M. (2018). Fictionalism versus deflationism: A new look. Philosophical Studies, 175(2), 301-316.

Putnam, H. (1980). Models and reality. In: Putnam (1983) (pp. 1-25). Originally published in Journal of Symbolic Logic 45(3), 464-482.

Putnam, H. (1983). Realism and reason philosophical papers (Vol. 3). Cambridge: Cambridge University Press.

Quine, W. O. (1960). Word and object. Cambridge: The MIT Press.

Raab, J. (2020/08). Easy ontology and conservativeness. Manuscript, August 2020.

Suppes, P. (1957). Introduction to logic. New York: Van Nostrand Reinhold Company.

Thomasson, A. L. (2007). Ordinary objects. Oxford: Oxford University Press.

Thomasson, A. L. (2008). Existence questions. Philosophical Studies, 141(1), 63-78.

Thomasson, A. L. (2009a). The easy approach to ontology. Axiomathes, 19, 1-15.

Thomasson, A. L. (2009b). Answerable and unanswerable questions. In: Chalmers \& Manley \& Wasserman (2009) (pp. 444-471).

Thomasson, A. L. (2013). Fictionalism versus deflationism. Mind, 122(488), 1023-1051.

Thomasson, A. L. (2014). Quizzical ontology and easy ontology. The Journal of Philosophy, 111(9/10), 502-528.

Thomasson, A. L. (2015). Ontology made easy. Oxford: Oxford University Press.

Thomasson, A. L. (2016). Carnap and the prospects for easy ontology. In: Blatti \& LaPointe (2016) (pp. 122-144).

Thomasson, A. L. (2017). Why we should still take it easy. Mind, 126(503), 769-779.

Yablo, S. (1998). Does ontology rest on a mistake? Proceedings of the Aristotelian Society, Supplementary, 72, 229-261.

Publisher's Note Springer Nature remains neutral with regard to jurisdictional claims in published maps and institutional affiliations. 